\title{
Dimers of Acetic Acid in Helium Nanodroplets
}

Julia A. Davies, ${ }^{\dagger}$ Magnus W. D. Hanson-Heine, ${ }^{+}$Nicholas A. Besley,,+ Andrew Shirley, ${ }^{\dagger}$ James Trowers, ${ }^{\dagger}$ Shengfu Yang, ${ }^{\dagger}$ and Andrew M. Ellis* ${ }^{\dagger}$

$\dagger$ Department of Chemistry, University of Leicester, University Road, Leicester, LE1 7RH, United Kingdom

$\$$ School of Chemistry, University of Nottingham, University Park, Nottingham, NG7 2RD, United Kingdom

\section{Corresponding Author}

* E-mail: andrew.ellis@,1e.ac.uk

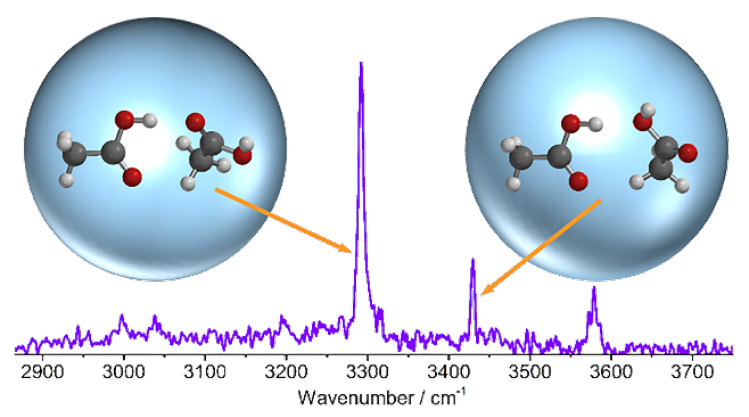

Electronic supplementary information contains additional results from quantum chemical calculations on the acetic acid monomer and selected dimers. 


\begin{abstract}
The structural arrangement of small carboxylic acid molecules in the liquid phase remains a controversial topic. Some studies indicate a dominance of the cyclic dimer that prevails in the gas phase, whilst other studies favor short fragments of the infinite catemer chains that are found in the crystalline phase. Furthermore, difficulties in preparing and probing size-selected catemer segments have resulted in a lack of benchmark data upon which theoretical models of the condensed phases can be built. To address these issues, we have combined infrared spectroscopy and quantum chemical calculations to explore regions of the intermolecular potential energy surface associated with the formation of metastable dimer isomers. The $\mathrm{OH}$ stretching region of the spectrum shows that aggregation of acetic acid molecules inside liquid helium nanodroplets yields two distinct metastable dimers, whilst negligible signal is observed from the cyclic dimer that typically overwhelms this spectral region. We deduce that the most abundant isomer in superfluid helium has one strong $\mathrm{O}-\mathrm{H} \cdots \mathrm{O}=\mathrm{C}$ and one weak $\mathrm{C}-\mathrm{H} \cdots \mathrm{O}=\mathrm{C}$ hydrogen bond. Since this bonding motif is common to the dimeric repeating unit of the catemer, it is of fundamental importance for understanding intermolecular interactions in the condensed phases of small carboxylic acids.
\end{abstract}




\section{Introduction}

Dimers of carboxylic acids are classic examples of hydrogen bonded structures. They are used as model systems to gain an understanding of proton donor-acceptor interactions, which are important in structural biology, molecular recognition and polymer science. ${ }^{1-5}$ The lowest energy structure of carboxylic acid homodimers has two strong and equivalent $\mathrm{O}-\mathrm{H} \cdots \mathrm{O}=\mathrm{C}$ hydrogen bonds, which yield the locally cyclic arrangement illustrated in Figure 1(a) for acetic acid (AA), and this cyclic structure is prevalent in the gas phase. ${ }^{6,7}$ However, the crystalline state of AA is dominated by catemer chains containing one strong $\mathrm{O}-\mathrm{H} \cdots \mathrm{O}=\mathrm{C}$ and one weak $\mathrm{C}-\mathrm{H} \cdots \mathrm{O}=\mathrm{C}$ bond between adjacent molecules, as shown in Figure 1(b), and the formation of extended structures is facilitated by weak $\mathrm{C}-\mathrm{H} \cdots \mathrm{O}$ bonds between the catemer chains. ${ }^{8,9}$

(a)

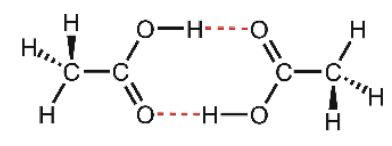

(c)
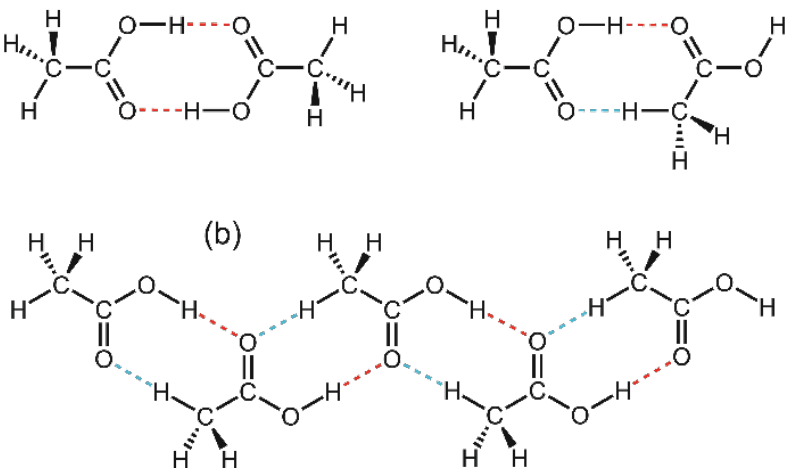

Figure 1. Selected hydrogen-bonded structures for trans acetic acid: (a) cyclic dimer, (b) a section of the catemer observed in the crystalline state, and (c) a metastable dimer. The red and pale blue dashed lines represent strong $\mathrm{O}-\mathrm{H} \cdots \mathrm{O}=\mathrm{C}$ and weak $\mathrm{C}-\mathrm{H} \cdots \mathrm{O}=\mathrm{C}$ hydrogen bonds, respectively.

The structure of the liquid phase is still the subject of controversy, with some studies indicating a preponderance of cyclic dimers ${ }^{10,11}$ whilst others favor segments of catemer chains, ${ }^{12,13}$ potentially as short as the trimer. ${ }^{14}$ Despite its important role as the repeating unit of the catemer structure, relatively little is known about the metastable AA dimer shown in Figure 1(c). In addition, even though the initial steps involved in AA crystallization remain unclear, this dimer could potentially 
act as a precursor to catemer formation and crystal growth. ${ }^{15}$ For these reasons, this metastable dimer is the primary focus of the present work.

Whilst techniques such as neutron and X-ray diffraction are valuable for determining intermolecular separations in the condensed phases, infrared (IR) spectroscopy is sensitive to the type and strength of intermolecular interactions that are fundamental to the formation of extended structures. For example, when a molecule containing an $\mathrm{OH}$ group forms a hydrogen bond of the type $\mathrm{O}-\mathrm{H} \cdots \mathrm{X}$, this weakens the $\mathrm{O}-\mathrm{H}$ bond and can result in a substantial red shift of the $\mathrm{OH}$ stretching frequency and an increase in the absorption strength. ${ }^{16,17}$

For small clusters at low temperature, IR spectra typically contain well defined peaks enabling definitive assignments to be made through comparison with quantum chemical calculations. However, for more complex systems, the spectral profile can be broad and relatively featureless, leading to difficulties in interpreting the spectrum; this is the case for solid and liquid AA. ${ }^{18}$ Nevertheless, insight into the hydrogen bonding interactions can be gained by gradually increasing the system complexity, for example, by starting with the dimeric unit of the catemer structure and adding one molecule at a time. This "bottom up" approach can also help in the development of theoretical models by providing valuable benchmarks.

The $\mathrm{OH}$ stretching region of the IR spectrum for the cyclic dimer of AA (Figure 1(a)) has been well studied and experiments have shown that, instead of a single feature corresponding to the antisymmetric $\mathrm{OH}$ stretching mode, a very broad and partly structured absorption envelope extends across $\sim 2500-3400 \mathrm{~cm}^{-1}$ with an intensity maximum at $\sim 2930 \mathrm{~cm}^{-1}$ for a jet-cooled gas phase sample. ${ }^{19-22}$ The spectral complexity has been the subject of many theoretical studies and is attributed to a variety of anharmonic coupling mechanisms. ${ }^{23-26}$ By comparison, little is known about other dimeric isomers such as that shown in Figure 1(c) and their study is hampered by (i) spectral congestion and weak signal intensities in the gas phase, ${ }^{20,27}$ (ii) large frequency shifts due to vibrational perturbation when studied in solid matrices ${ }^{28-30}$ and (iii) the lack of accurate calculations of vibrational frequencies for comparison with experiment until recently. ${ }^{30}$ Such problems can potentially be resolved by preparing high purity samples of metastable dimers in low perturbation environments, such as inside superfluid helium nanodroplets. 
It is well established that cluster formation inside helium nanodroplets can lead to the creation of isomers other than the global energy minimum, e.g., the cyclic hexamer of water, ${ }^{31}$ linear chains of $(\mathrm{HCN})_{n}$ where $n=4$ to $7,{ }^{32}$ and the linear OCS trimer. ${ }^{33}$ Furthermore, vibrational perturbation of the dopants is known to be small compared with solid matrix environments, enabling more reliable comparison with quantum chemical calculations. ${ }^{34}$ In an earlier IR spectroscopic study of formic acid (FA) dimers in helium nanodroplets, it was deduced that a single metastable dimer structure formed instead of the lowest energy cyclic isomer. ${ }^{35}$ However, the absorption strength was reported to be exceptionally weak in the hydrogen-bonded $\mathrm{OH}$ stretching region, ${ }^{35}$ despite predictions of strong bands from ab initio calculations. ${ }^{36}$ The reason for this disparity is unknown and it is not a priori clear whether similar isomeric selectivity will be observed for AA dimers because the balance of intermolecular forces is expected to be far more subtle for AA than FA. ${ }^{29}$

In this work, we observe the spectral signature of two metastable dimers of AA, which are formed inside superfluid helium at $\sim 0.37 \mathrm{~K}$, with no detectable contribution from the lowest energy cyclic dimer that dominates in the gas phase. We have performed spectroscopic characterization in the $\mathrm{OH}$ stretching region and compared our measurements with ab initio calculations. Properties relating to the $\mathrm{O}-\mathrm{H} \cdots \mathrm{O}=\mathrm{C}, \mathrm{C}-\mathrm{H} \cdots \mathrm{O}=\mathrm{C}$ and $\mathrm{O}-\mathrm{H} \cdots \mathrm{O}-\mathrm{H}$ hydrogen bonding interactions are reported, including calculated intermolecular bond lengths and interaction energies for the observed metastable dimers.

\section{Methodology}

\subsection{Experimental}

Experiments were performed using a previously described helium nanodroplet instrument. ${ }^{37-39}$ The nanodroplets were prepared by expanding helium gas at high pressure ( 32 bar) through the $5 \mu \mathrm{m}$ diameter aperture of a cooled nozzle $(16 \mathrm{~K})$, resulting in a mean droplet size of $\sim 5000$ helium atoms. ${ }^{34}$ The resulting continuous beam of droplets passed through a skimmer and into a cell containing AA vapor, where they could pick up one or more AA molecules. After leaving the pickup cell, the doped droplets were overlapped with a counter-propagating tunable IR laser beam 
from a nanosecond optical parametric amplifier (LaserVision) operating at a repetition rate of 10 $\mathrm{Hz}$, with bandwidth of $\sim 4 \mathrm{~cm}^{-1}$ and an incident pulse energy of $\sim 11 \mathrm{~mJ}$.

Upon reaching a quadrupole mass spectrometer (Extrel, MAX-1000), the doped droplets were ionized via $90 \mathrm{eV}$ electron impact and the fragment ions were detected. The mass spectrometer was operated in two different modes. The first permitted the measurement of a mass spectrum over a wide range of ion masses, whilst the second enabled the selection of ions with a specific massto-charge ratio, $\mathrm{m} / \mathrm{z}$. Using the latter operating mode, the ion signal was accumulated using a gated photon counter (Stanford Research Systems, SR400) and IR spectra were obtained by measuring the change in ion signal as the laser wavelength was scanned. If the wavelength matches an absorption transition of the dopant, the energy absorbed is converted to heat and causes the droplet to shrink via evaporation of helium atoms. Consequently, IR absorption is registered as a depletion in the ion signal of a selected mass channel.

The AA vapor was obtained from a sample of glacial acetic acid at room temperature, which was bled into the pickup cell through a needle valve, enabling a controlled partial pressure to be achieved. In order to confirm the size of the dopant cluster responsible for each IR absorption feature, the dependence of the pickup cell pressure (PUCP) on the ion depletion signal was measured for selected mass channels. The resulting PUCP curves were subsequently compared with the normalized Poisson distributions expected for a statistical pick up of AA monomers, dimers and trimers (see later). When recording IR spectra over an extended frequency range, an AA partial pressure of $8 \times 10^{-6}$ mbar was chosen, which corresponds to an average pick up of one AA molecule per droplet. This low partial pressure reduces the probability that a droplet will acquire three or more AA molecules and therefore the resulting IR spectra are dominated by peaks associated with the monomer and dimers.

\subsection{Computational methods}

Quantum chemical calculations were performed using the Q-Chem software package. ${ }^{40}$ Due to its relatively low cost, density functional theory (DFT) was used to search for equilibrium structures of AA dimers, which correspond to minima on the intermolecular potential energy surface. In order to search through a wide range of possible geometries, a total of 100 different initial configurations were generated by randomly performing 30 rotational and translational moves. In a translational 
move the molecule is shifted by a random distance between 0 and the maximum displacement of $5 \mathrm{a}_{0}$, where $\mathrm{a}_{0}=1$ bohr, in each of the $x, y$ and $z$ directions. In a rotational move the molecule is rotated by a random amount between $0^{\circ}$ and $180^{\circ}$ about a randomly selected inertial axis. A move is rejected if the molecule moves within 0.3 a o of another molecule or goes outside a cubic box of side $40 \mathrm{a}_{0}$. Only the lowest energy trans conformer of each monomeric unit was considered for the initial configurations, which were optimized without constraint using DFT at the M06/6-31+G(d) level with the SG-1 integration grid. The differentiated species were then re-optimized at the M06/6-311++G(d,p) level of theory, with an EML-(150,770) integration grid.

For comparison with experiment, geometry optimization and frequency calculations were subsequently performed using second-order Møller-Plesset perturbation theory (MP2) in combination with the 6-311(2+,2+) $\mathrm{G}(2 \mathrm{~d}, 2 \mathrm{p})$ basis set. These calculations utilized the structures found from the preceding DFT calculations as a starting point. A zero-point energy (ZPE) correction was applied to the interaction energies of the dimers and the harmonic frequencies were scaled by a factor of 0.9463 . The latter value was deduced by comparing the calculated $\mathrm{OH}$ stretching frequency for the AA monomer with the known experimental value of $3583 \mathrm{~cm}^{-1}$ in the gas phase. ${ }^{41}$ This mode-specific scaling factor provides a correction for the neglect of anharmonicity, in addition to addressing residual deficiencies in the electronic structure calculations. Anharmonic frequency calculations were not attempted because they are expensive for systems as large as an AA dimer using methods such as MP2 and studies suggest that the gain in accuracy over scaled harmonic frequency calculations can be quite modest. ${ }^{42,43}$

\section{Results and discussion}

\subsection{Experimental IR depletion spectra}

Figure 2(a) shows a mass spectrum, which is dominated by three peaks corresponding to ion mass channels $m / z$ 43, 45 and 61 . Because the relative molecular mass for the AA monomer is 60 , the ion signals at $m / z 43$ and 45 can originate from fragmentation of the ionized AA neutral monomer and from larger clusters, whereas the origin of the $m / z 61$ signal can only come from AA dimers and larger clusters. Even though a range of parent cluster sizes can contribute to each ion mass 
channel, the channels primarily derived from the parent dimer can be determined from analysis of the IR spectra and PUCP curves, as detailed below.
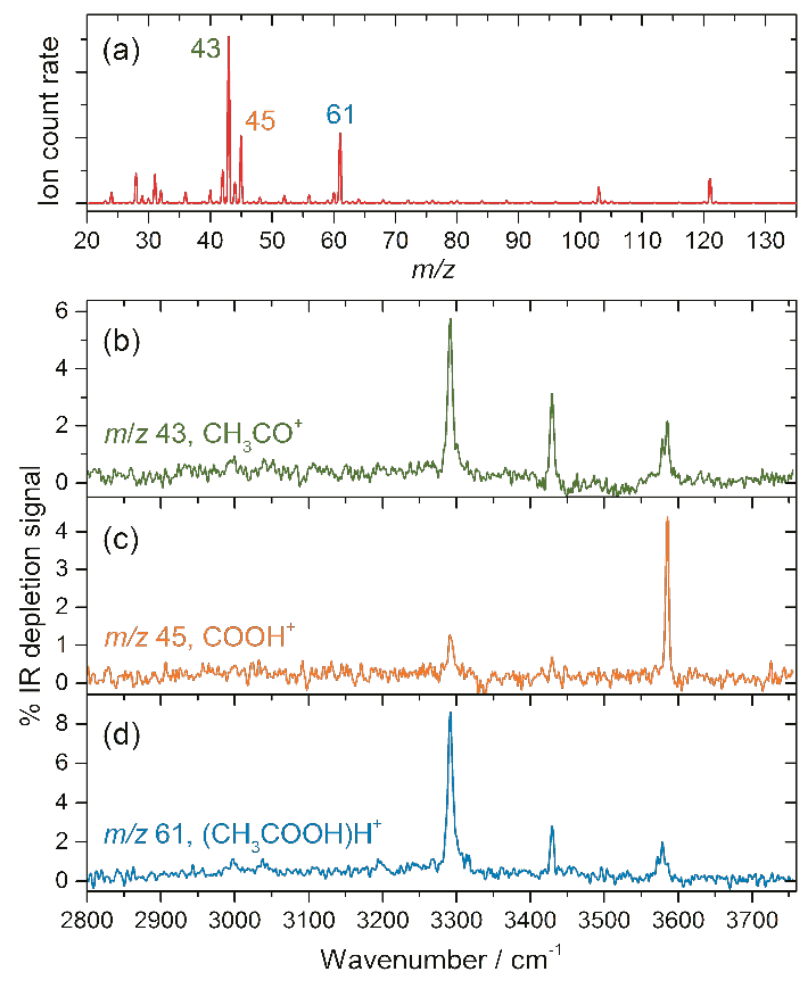

Figure 2. (a) Mass spectrum obtained from electron ionization of helium nanodroplets containing acetic acid. Beneath the mass spectrum are IR spectra recorded by monitoring the depletion in signal for fragment ions at (b) $m / z 43$, (c) $m / z 45$ and (d) $m / z$ 61. For all spectra, the average number of acetic acid molecules added per droplet is one.

In Figure 2(b) to (d), IR spectra recorded in the $\mathrm{OH}$ stretching region from 2800 to $3760 \mathrm{~cm}^{-1}$ for each of the three dominant ion mass channels are presented. The IR spectrum recorded for the $\mathrm{COOH}^{+}$fragment ion at $\mathrm{m} / \mathrm{z} 45$ in Figure 2(c) is dominated by a sharp peak at $3585 \mathrm{~cm}^{-1}$. Because the IR absorption event occurs prior to ionization, the spectral peak is assigned to a vibrational mode of the neutral dopant rather than the detected ion. Previous experimental gas phase studies have reported that the free $\mathrm{OH}$ stretching mode for the AA monomer lies at $3583 \mathrm{~cm}^{-1},^{41}$ and therefore the IR peak that we observe at $3585 \mathrm{~cm}^{-1}$ is assigned to the same vibrational transition. 
We note that this transition derives from the trans conformation, ${ }^{20}$ which has an energy $\sim 23 \mathrm{~kJ}$ mol $^{-1}$ lower than the cis conformer. ${ }^{44}$

In Figure 3, two distinct sets of PUCP curves are presented for the spectral feature at $3585 \mathrm{~cm}^{-1}$; one from the ion channel with $m / z 45$ and the other for $m / z$ 43. Both sets show a similar pressure dependence, indicating that there are at least two fragmentation pathways following electron ionization of the AA monomer, i.e., fragmentation to $\mathrm{COOH}^{+}$at $m / z 45$ and to $\mathrm{CH}_{3} \mathrm{CO}^{+}$at $m / z 43$. The IR feature at $3585 \mathrm{~cm}^{-1}$ for the latter mass channel is observed in Figure 2(b) as the higher frequency component of a partially resolved doublet.

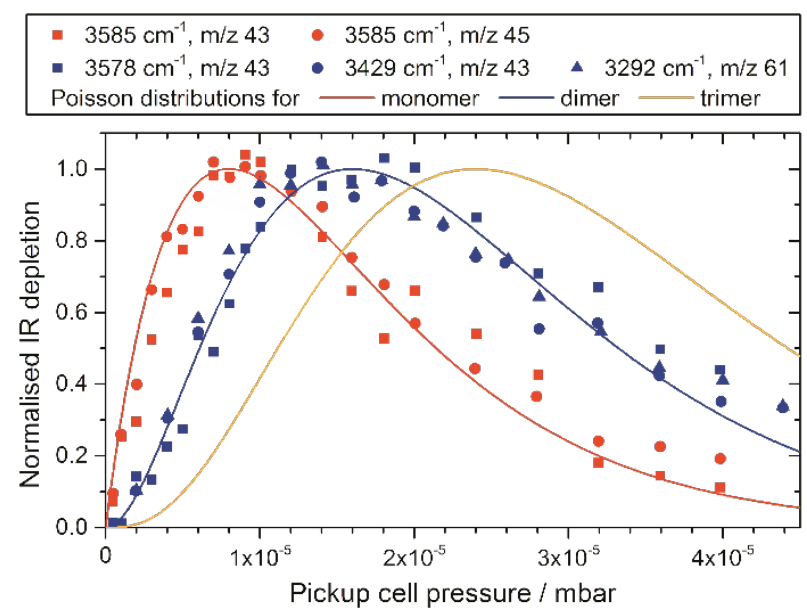

Figure 3. Pickup cell pressure curves obtained by measuring the depletion in ion signal at fixed IR wavelengths corresponding to the dominant spectral features. Data are shown for fragment ions with $m / z 43,45$ and 61 . The solid lines show the expected Poisson distribution curves for the acetic acid monomer, dimer and trimer.

Strong similarities are observed for the IR spectra in Figures 2(b) and (d), which were measured by detecting $\mathrm{CH}_{3} \mathrm{CO}^{+}$and $\left(\mathrm{CH}_{3} \mathrm{COOH}\right) \mathrm{H}^{+}$ions at $m / z 43$ and 61, respectively, and the remarkable finding here is the simplicity of the spectra. Three strong and reasonably sharp bands at 3292, 3429 and $3578 \mathrm{~cm}^{-1}$ are common to both mass channels and are assigned to $\mathrm{OH}$ stretching modes. As 
illustrated in Figure 3, all three of these bands show a pressure-dependence in good agreement with the Poisson distribution for the neutral AA dimer.

Even though a small IR depletion signal is observed below $3280 \mathrm{~cm}^{-1}$ for the $m / z 61$ mass channel (see Figure 2(d)), the absence of a local maximum in signal at $\sim 2930 \mathrm{~cm}^{-1}$ indicates that this structure does not originate from the cyclic dimer. Instead, we assign the weak features at $~ 3000$ and $\sim 3040 \mathrm{~cm}^{-1}$ to $\mathrm{CH}$ stretching modes of metastable AA dimers and the minor feature at $\sim 3200$ $\mathrm{cm}^{-1}$ to an $\mathrm{OH}$ stretching mode of a trimer that will be the subject of future work. A very weak and broad spectral profile is also observed below $3280 \mathrm{~cm}^{-1}$ and we preliminarily assign this to AA clusters larger than the dimer. The absence of signal from the cyclic dimer is in stark contrast to previous IR spectroscopy studies in both the gas phase and in argon matrices, suggesting that either the cyclic dimer is not formed inside helium nanodroplets or else the population is particularly low. This is fortuitous since the lack of spectral contamination from the cyclic dimer allows for a more detailed study of metastable AA dimers.

In the present work, the dimer band at $3578 \mathrm{~cm}^{-1}$ has a small red-shift from the free $\mathrm{OH}$ stretch of the AA monomer at $3585 \mathrm{~cm}^{-1}$ and must therefore originate from a free $\mathrm{OH}$ group of an AA dimer. Because both $\mathrm{OH}$ groups in the lowest energy cyclic dimer are strongly bound, as illustrated in Figure 1(a), the observation of a free $\mathrm{OH}$ band confirms that metastable dimers are formed. The other IR bands at 3292 and $3429 \mathrm{~cm}^{-1}$ are substantially red-shifted from the free $\mathrm{OH}$ stretching region and are therefore assigned to $\mathrm{OH}$ groups involved in hydrogen bonding. We note that if these two bands came from the same dimer structure then their relative intensities would be the same regardless of which mass channel was used for detection. However, what we actually see is a difference in relative band intensities when spectra are recorded in the $m / z 43$ and 61 mass channels, showing that the bands at 3292 and $3429 \mathrm{~cm}^{-1}$ are from different isomers. The implication here is that these isomers have different fragmentation branching ratios on ionization, a fact we attribute to differences in their structures.

\subsection{Assignment of dimer isomers}

In order to deduce the geometric structures of the AA dimers created inside helium nanodroplets, we have performed quantum chemical calculations. In making a comparison between theory and experiment, it is worth noting that very small vibrational shifts are expected for molecules encased 
in superfluid helium, with most known cases showing shifts of $<2 \mathrm{~cm}^{-1}$ when compared with the gas phase. ${ }^{34}$ Furthermore, the apparent absence of anharmonic coupling between the $\mathrm{OH}$ stretching modes and lower energy vibrational modes, which we infer from the sharpness of the IR bands in Figure 3, should make for a reasonably straightforward comparison between the calculated scaled harmonic frequencies and experimental values.

Our calculations began with a random search procedure, which yielded 11 unique dimer structures with energies within $70 \mathrm{~kJ} \mathrm{~mol}^{-1}$ of the global minimum energy structure. Examination of the vibrational frequencies and intensities revealed that the seven highest energy dimer structures have scaled harmonic IR frequencies $>3570 \mathrm{~cm}^{-1}$ and low intensities of $\leq 120 \mathrm{~km} \mathrm{~mol}^{-1}$ for both OH stretching modes. This indicates an absence of strong intermolecular bonds involving an $\mathrm{OH}$ group. Therefore, these seven structures are not suitable candidates for assignment to the experimental IR peaks at 3292 and $3429 \mathrm{~cm}^{-1}$ and will not be considered further.

In addition to the four remaining dimer structures that were found by our search procedure, two other structures, which were identified in previous theoretical studies, ${ }^{7,30,46}$ were also considered as possible candidates because they contain hydrogen-bonded $\mathrm{OH}$ groups. To identify these structures through our search procedure would have required expanding the search beyond the 100 initial structures considered. However, as these structures are already known from elsewhere we included them directly. We therefore have six structures, labelled D1 to D6, that we will focus on from here onwards. These structures are labelled in order of increasing energy so that D1 is the cyclic dimer (the global potential energy minimum). Isomers D4 and D5 are those taken from previous studies. Our calculations at the MP2/6-311(2+,2+)G(2d,2p) level of theory find that one of these isomers, D5, has an imaginary value for the lowest frequency vibration. The presence of this imaginary frequency is dependent on the methodology used, since no imaginary frequencies were observed for DFT calculations and MP2 calculations with a smaller $6-31+\mathrm{G}^{*}$ basis set. The higher level calculations suggest that D5 is a transition state where the imaginary mode corresponds to a twisting of the AA molecules around the hydrogen bond, which takes the structure towards D4. 
In Figure 4, the experimental IR spectrum for the $\mathrm{m} / \mathrm{z} 43$ channel is reproduced. Also shown are the structures for isomers D1 to D6, along with the IR spectra predicted from our MP2 calculations. The comparison between theory and experiment begins with the most strongly red-shifted experimental feature observed at $3292 \mathrm{~cm}^{-1}$ in Figure 4(a). This peak shows close agreement with the $v(\mathrm{OH}) \cdots \mathrm{O}=\mathrm{C}$ stretching mode for dimer $\mathrm{D} 3$ in the calculated spectra in Figure 4(b). The substantial redshift relative to the AA monomer is due to the strength of the $\mathrm{O}-\mathrm{H} \cdots \mathrm{O}=\mathrm{C}$ hydrogen bond. However, because D3 only contains one such bond, as illustrated by the calculated structure shown in Figure 4(c), the redshift is substantially smaller than that predicted for the cyclic dimer, D1. The assignment to D3 follows because all other calculated frequencies are $>30 \mathrm{~cm}^{-1}$ from the experimental value. A low intensity feature at $3575 \mathrm{~cm}^{-1}$ is also predicted for D3, which almost exactly matches the experimental frequency for the free $\mathrm{OH}$ stretch in the dimer.
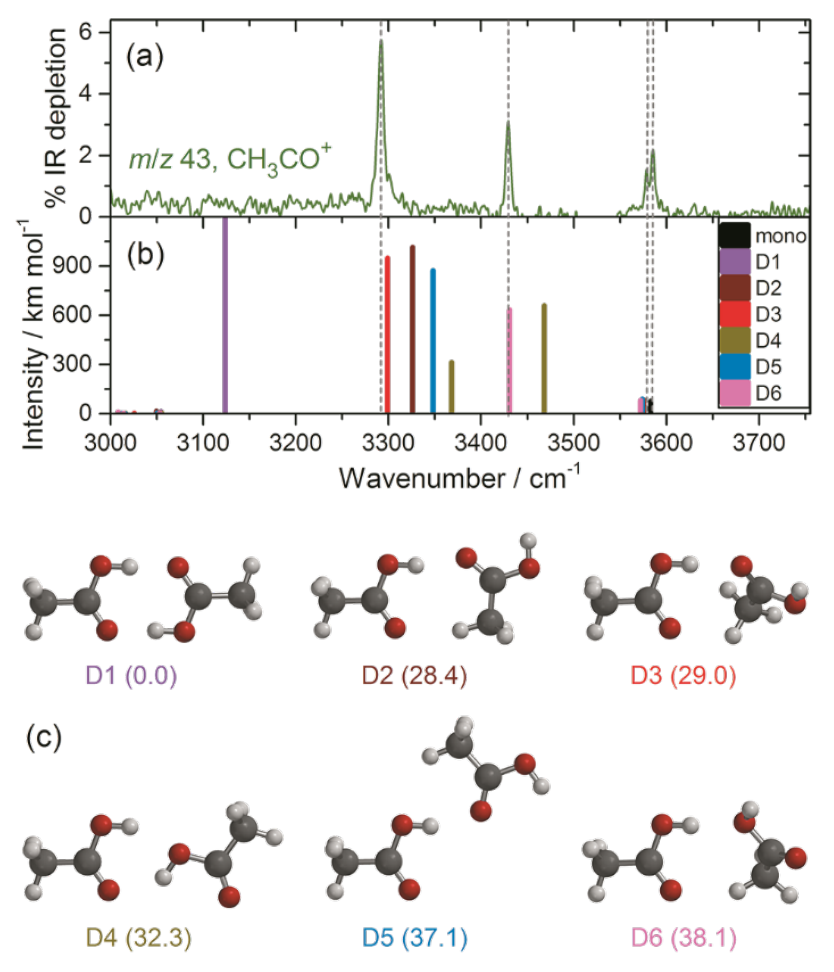

Figure 4. Comparison between (a) an IR depletion spectrum measured by monitoring ions at $\mathrm{m} / \mathrm{z}$ 43 and (b) scaled harmonic MP2 calculations for the acetic acid monomer and the six lowest energy dimers, D1 to D6, whose structures are shown in (c) along with the ZPE-corrected relative total 
energies $\left(\mathrm{kJ} \mathrm{mol}^{-1}\right)$ in parentheses. The dashed vertical lines indicate the centres of the experimental IR bands.

The band at $3429 \mathrm{~cm}^{-1}$ in Figure 4(a) is indicative of a second isomer of the AA dimer. This band lies to the blue of the bonded $\mathrm{OH}$ stretch for $\mathrm{D} 3$ and so must originate from an isomer with a weaker hydrogen bond involving a hydroxyl group. Assignment of this isomer is again straightforward following comparison with the calculated spectra in Figure 4(b) and we assign it to stretching of the proton donor $\mathrm{OH}$ for dimer D6, whose calculated structure is also shown in Figure 4(c).

Selected results from the MP2 calculations are presented in Table 1, confirming that the scaled vibrational frequencies for the bonded $\mathrm{OH}$ stretching modes for dimers D3 and D6 are in excellent agreement, to within $7 \mathrm{~cm}^{-1}$, with the experimental bands. Both of these structures were successfully identified by our search procedure using DFT and the calculated vibrational modes were determined to be real at all levels of theory used. We therefore conclude that dimer structures D3 and D6 are formed inside helium nanodroplets.

Table 1. Selected results from quantum chemical calculations (MP2) for the six lowest energy dimers of acetic acid along with the experimental IR band positions recorded in this study.

\begin{tabular}{|c|c|c|c|c|c|}
\hline $\begin{array}{l}\text { Dimer } \\
\text { (symmetry) }\end{array}$ & $\begin{array}{l}\text { Energy }^{a} \\
/ \mathrm{kJ} \mathrm{mol}^{-1}\end{array}$ & \multicolumn{2}{|c|}{ Calculated OH stretches ${ }^{b} / \mathrm{cm}^{-1}$} & $\begin{array}{l}\text { Experimental OH } \\
\text { stretches } / \mathrm{cm}^{-1}\end{array}$ & $\begin{array}{l}\text { Dipole moment } \\
\text { /Debye }\end{array}$ \\
\hline $\mathrm{D} 1\left(\mathrm{C}_{2 \mathrm{~h}}\right)$ & -63.1 & $3031(0)$ & $3124(2970)$ & & 0.00 \\
\hline $\mathrm{D} 2\left(\mathrm{C}_{\mathrm{s}}\right)$ & -34.7 & $3326(1013)$ & $3576(83)$ & & 1.32 \\
\hline D3 $\left(\mathrm{C}_{1}\right)$ & -34.1 & 3299 (947) & $3575(85)$ & 3292,3578 & 1.07 \\
\hline $\mathrm{D} 4^{c}\left(\mathrm{C}_{\mathrm{s}}\right)$ & -30.8 & 3368 (312) & $3468(658)$ & & 3.29 \\
\hline $\mathrm{D}^{c, d}\left(\mathrm{C}_{\mathrm{s}}\right)$ & -26.0 & $3348(871)$ & 3574 (88) & & 3.66 \\
\hline D6 $\left(\mathrm{C}_{1}\right)$ & -25.0 & $3431(633)$ & $3573(82)$ & 3429,3578 & 2.24 \\
\hline
\end{tabular}

${ }^{a}$ ZPE-corrected interaction energy given relative to the summed energies of two monomers.

${ }^{b}$ Scaled harmonic vibrational frequencies for the two $\mathrm{OH}$ stretching vibrations in the dimer. The scaling factor is 0.9463 . The quantities in parentheses are the IR intensities expressed in $\mathrm{km} \mathrm{mol}^{-1}$. 
${ }^{c}$ These dimers were not found by our initial random search procedure for stable structures.

${ }^{d}$ Predicted to be a transition state in our calculations.

The calculated interaction energies shown in Table 1 indicate relatively small energy differences between the pairs of isomers D2 and D3, and D5 and D6. MP2 calculations are known to have a tendency to overestimate dispersion interactions ${ }^{47}$ so we have performed additional calculations at the coupled cluster level (CCSD/aug-cc-pVDZ) using the geometries determined using MP2 and these calculations find the same the energy ordering for the isomers.

The relative populations of the two AA isomers prepared in helium nanodroplets can be estimated from the peak intensities in the experimental spectra and the calculated IR band strengths (Table 1). Assuming that the population of other dimers is negligible, we determine that $\sim 60 \%$ of the dimers have structure D3 whilst the remaining $\sim 40 \%$ have structure D6. These dimers have different hydrogen bonding motifs, namely $\mathrm{O}-\mathrm{H} \cdots \mathrm{O}=\mathrm{C}$ and $\mathrm{C}-\mathrm{H} \cdots \mathrm{O}=\mathrm{C}$ for $\mathrm{D} 3$ and $\mathrm{O}-\mathrm{H} \cdots \mathrm{O}-\mathrm{H}$ and $\mathrm{C}-\mathrm{H} \cdots \mathrm{O}=\mathrm{C}$ for $\mathrm{D} 6$, which leads to significant differences in the intermolecular bond lengths shown in Table 2 and in Figure 5. However, the most striking feature is the remarkable similarity in the relative positions of the atoms in the two structures; they are almost identical apart from the positions of the hydrogen atoms (see Figure 4(c)). Interestingly, the difference in location of the carboxyl hydrogen leads to a large change in the total dipole moment, with the value for D6 being over twice that for D3 (see Table 1). An alternative way of comparing the structures of the D3 and D6 isomers is to note that they are related by a $\sim 180^{\circ}$ rotation of one of the monomers about the $\mathrm{C}-\mathrm{C}$ bond, resulting in a change of the hydrogen bond acceptor from the $\mathrm{OH}$ group in D6 to the carbonyl group in D3.

In Table 2 it can be seen that $\mathrm{D} 2$ and $\mathrm{D} 3$ possess the same bonding motif, which is identical to that for the dimeric unit of the catemer chain (see Figure 1(b) and (c)). It is therefore perhaps surprising that only one of the dimers is observed inside helium nanodroplets, but our results are decisive in this matter. Our calculations also show that $\mathrm{D} 2$ and $\mathrm{D} 3$ have similar $\mathrm{O}-\mathrm{H} \cdots \mathrm{O}=\mathrm{C}$ bond lengths. However, the $\mathrm{C}-\mathrm{H} \cdots \mathrm{O}=\mathrm{C}$ bond length for $\mathrm{D} 2$ is shorter by $\sim 10 \%$, which can be attributed to the planar geometry shown in Figure 5. The shorter intermolecular bond length also results in a slightly lower interaction energy (see Table 1). This is an interesting observation because $\mathrm{C}-\mathrm{H} \cdots \mathrm{O}=\mathrm{C}$ bonds are approximately an order of magnitude weaker than $\mathrm{O}-\mathrm{H} \cdots \mathrm{O}=\mathrm{C}$ bonds and it would therefore be easy to underestimate their influence. However it is well known that weak $\mathrm{C}-\mathrm{H} \cdots \mathrm{O}$ 
bonds play an important role in stabilizing the AA crystalline structure, ${ }^{8,9}$ and the present calculations provide a tangible example of this behavior.

Table 2. Hydrogen bond lengths obtained from MP2 calculations for the six lowest energy metastable dimers of acetic acid.

\begin{tabular}{lllll}
\hline $\begin{array}{l}\text { Dimer } \\
\text { (symmetry })\end{array}$ & H-bond type & $\begin{array}{l}\text { Bond } \\
\text { length } / \AA\end{array}$ & H-bond type & $\begin{array}{l}\text { Bond } \\
\text { length } / \AA\end{array}$ \\
\hline $\mathrm{D} 1\left(\mathrm{C}_{2 \mathrm{~h}}\right)$ & $\mathrm{O}-\mathrm{H} \cdots \mathrm{O}=\mathrm{C}$ & 1.677 & $\mathrm{O}-\mathrm{H} \cdots \mathrm{O}=\mathrm{C}$ & 1.677 \\
$\mathrm{D} 2\left(\mathrm{C}_{\mathrm{s}}\right)$ & $\mathrm{O}-\mathrm{H} \cdots \mathrm{O}=\mathrm{C}$ & 1.798 & $\mathrm{C}-\mathrm{H} \cdots \mathrm{O}=\mathrm{C}$ & 2.294 \\
$\mathrm{D} 3\left(\mathrm{C}_{1}\right)$ & $\mathrm{O}-\mathrm{H} \cdots \mathrm{O}=\mathrm{C}$ & 1.778 & $\mathrm{C}-\mathrm{H} \cdots \mathrm{O}=\mathrm{C}$ & 2.542 \\
$\mathrm{D} 4\left(\mathrm{C}_{\mathrm{s}}\right)$ & $\mathrm{O}-\mathrm{H} \cdots \mathrm{O}=\mathrm{C}$ & 1.921 & $\mathrm{O}-\mathrm{H} \cdots \mathrm{O}-\mathrm{H}$ & 1.932 \\
$\mathrm{D} 5\left(\mathrm{C}_{\mathrm{s}}\right)$ & $\mathrm{O}-\mathrm{H} \cdots \mathrm{O}=\mathrm{C}$ & 1.831 & $\mathrm{C}-\mathrm{H} \cdots \mathrm{O}-\mathrm{H}$ & 2.597 \\
$\mathrm{D} 6\left(\mathrm{C}_{1}\right)$ & $\mathrm{O}-\mathrm{H} \cdots \mathrm{O}-\mathrm{H}$ & 1.894 & $\mathrm{C}-\mathrm{H} \cdots \mathrm{O}=\mathrm{C}$ & 2.349 \\
\hline
\end{tabular}
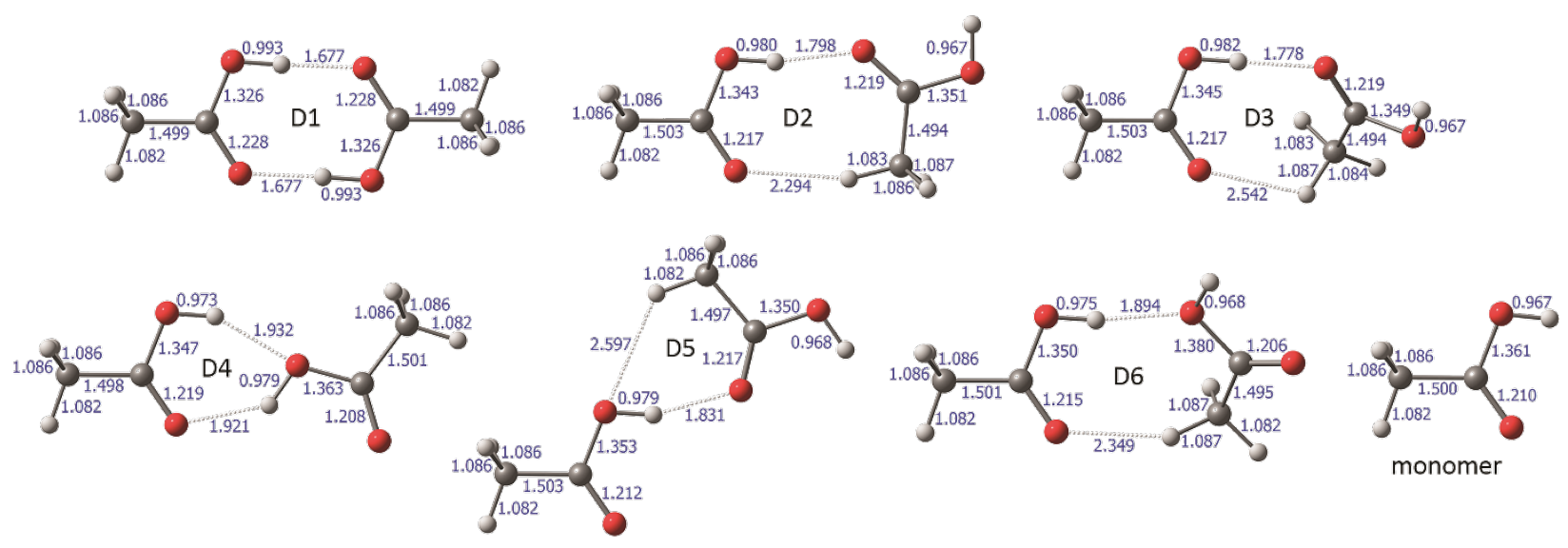
Figure 5. Calculated structures for the six lowest energy acetic acid dimers and the monomer. The intramolecular and hydrogen bond lengths are indicated.

\subsection{Comparison with previous experiments}

The IR spectrum for AA inside helium nanodroplets has not been measured previously. Therefore our results are compared with those obtained using cold samples of AA in alternative environments, such as in a jet-cooled gas or in matrix isolation. In Table 3, a comparison of the experimental band positions for the bonded $\mathrm{OH}$ stretching modes of the two metastable isomers, D3 and D6, is shown. Suhm and co-workers determined that the jet-cooled IR spectrum of gaseous AA is dominated by a broad but structured absorption feature that is characteristic of the cyclic dimer. ${ }^{20,27}$ Additional signal was observed from a metastable dimer as a relatively weak but distinct shoulder at $\sim 3300 \mathrm{~cm}^{-1}$. This feature was assigned to the bonded $\mathrm{OH}$ stretching mode in a dimer structure with $\mathrm{C}_{1}$ symmetry and the same bonding motif as isomer D3, and is therefore consistent with the present work.

Table 3. Comparison of $\mathrm{OH}$ stretching frequencies $\left(\mathrm{in}^{-1}\right)$ for metastable acetic acid dimers with those from previous experimental studies.

\begin{tabular}{|c|c|c|c|c|c|c|}
\hline \multirow[b]{2}{*}{ Dimer } & \multicolumn{2}{|c|}{ This work } & \multicolumn{4}{|c|}{ Previous experimental studies } \\
\hline & $\begin{array}{c}\text { Helium } \\
\text { nanodroplet }\end{array}$ & $\begin{array}{c}\text { MP2 } \\
\text { calculations }\end{array}$ & $\begin{array}{c}\text { Gas phase } \\
\text { Ref. } 27\end{array}$ & $\begin{array}{l}\text { Ar matrix } \\
\text { Ref. } 28\end{array}$ & $\begin{array}{l}\text { Ar matrix } \\
\text { Ref. } 29\end{array}$ & $\begin{array}{c}\mathrm{N}_{2} \text { matrix } \\
\text { Ref. } 30\end{array}$ \\
\hline D3 & 3292 & 3299 & 3304 & $3250^{\mathrm{a}}$ & $3251.8^{\mathrm{a}}$ & 3293.3 \\
\hline D6 & 3429 & 3429 & - & $3416^{\mathrm{a}}$ & $3417.0^{\mathrm{a}}$ & - \\
\hline
\end{tabular}

${ }^{a}$ Reassigned to structure D3 or D6 in the present work.

An argon matrix isolation study at $20 \mathrm{~K}$ reported a broad peak centered at $3250 \mathrm{~cm}^{-1}$ and a low intensity peak at $3416 \mathrm{~cm}^{-1}$. These two peaks were assigned to $v(\mathrm{OH}) \cdots \mathrm{O}=\mathrm{C}$ and $v(\mathrm{OH}) \cdots \mathrm{O}-\mathrm{H}$ 
stretches in open dimers. ${ }^{28}$ Even though the dimer structures were not identified, these bond types are consistent with those in D3 and D6, respectively, which are the same dimers identified in our helium nanodroplet studies. However, because solid rare gas matrices typically lead to far stronger perturbations of the vibrational modes than superfluid helium, red-shifts of 42 and $13 \mathrm{~cm}^{-1}$ were observed relative to the present work. A comparison of the vibrational frequencies reported in the argon matrix work with the calculated values for D1 to D6, shown in Table 1, further confirms these assignments.

In a more recent argon matrix isolation study at a lower temperature of $10 \mathrm{~K}$, IR bands at 3252 and $3417 \mathrm{~cm}^{-1}$ were reported to appear, but only under certain pulse deposition conditions, and were attributed to metastable AA dimers. ${ }^{29}$ Unfortunately, the spectral evidence for these bands was not provided and so we are unable to comment on the band widths and relative intensities. Nevertheless, as shown in Table 3, the reported frequencies are almost identical to those from the earlier argon matrix study, indicating that the same metastable dimers were prepared in both cases. However, because the authors only considered one metastable dimer when making their assignments, they assigned the two observed IR peaks to the bonded and free $\mathrm{OH}$ stretching modes in dimer D2. We note that the latter assignment to a free $\mathrm{OH}$ stretch mode is unlikely because of the excessive red-shift relative to the monomer $\mathrm{OH}$ stretch frequency of $3564 \mathrm{~cm}^{-1}$ that was measured in the same study. Therefore, following a comparison with the calculated $\mathrm{OH}$ stretch frequencies for D1 to D6 in Table 1, we propose that the two peaks at 3252 and $3417 \mathrm{~cm}^{-1}$ should be reassigned to the bonded $\mathrm{OH}$ stretching modes of D3 and D6, respectively.

A recent study of AA dimers in a solid nitrogen matrix identified IR features arising from numerous metastable dimers, including several involving the cis-AA isomer. ${ }^{30}$ Following deposition of a mixture of gaseous AA and nitrogen at $8.9 \mathrm{~K}$, the authors used photoexcitation and/or annealing processes to generate metastable trans-trans dimers and trans-cis dimers, which were subsequently probed using FTIR spectroscopy. The experimental studies were complemented by a comprehensive set of calculations at the MP2/6-311++G(2d,2p) level of theory, which found nine stable trans-trans dimers, fourteen trans-cis dimers and six cis-cis dimers. However, the downside of using a nitrogen matrix is that the assignment of the observed IR peaks is complicated by (i) large vibrational frequency shifts due to strong perturbations by the nitrogen matrix and (ii) the potential for multiple peaks to be assigned to the same transition 
because of matrix site effects. For example, IR peaks at 3545 and $3554 \mathrm{~cm}^{-1}$ were both assigned to the $\mathrm{OH}$ stretching mode for the trans-AA monomer but in different matrix sites and these values are red-shifted by 38 and $29 \mathrm{~cm}^{-1}$, respectively, from the known gas phase value. Despite these complications, an IR peak at $3293 \mathrm{~cm}^{-1}$ was assigned to the bonded $\mathrm{OH}$ stretching mode of dimer D3. Surprisingly, given the expectation of a substantial matrix shift, this is in excellent agreement with the value of $3292 \mathrm{~cm}^{-1}$ obtained in our helium nanodroplet study. However, in spite of the large number of IR features observed in the matrix work, none were assigned to dimer D6.

Our review of previous studies allows us to deduce that dimers D3 and D6 are observed to coexist, not only inside helium nanodroplets, but also in argon matrices at temperatures of $\leq 20 \mathrm{~K}$, albeit at much lower abundance in the latter environment. In contrast, dimer D6 was not observed in a jet-cooled gas sample, even though D3 was clearly present. A potential explanation is that both D3 and D6 are initially formed under cooled conditions in all of these media, but only the helium nanodroplets and argon matrices are effective at trapping the higher energy structure. This in turn implies that isomerization can occur more readily from D6 to lower energy structures than from D3.

Furthermore, not only do we observe the pair of dimers D3 and D6 inside helium nanodroplets, but there is also a noted absence of signal from D1, D2, D4 and D5 in our IR spectra. This indicates that any residual internal energy, such as rotational, vibrational and translational energy, is rapidly quenched by the helium and therefore the dimers remain trapped in structures D3 and D6. In support of this conjecture, we note that temperature-dependent stabilization of AA metastable dimers has previously been measured in an argon matrix study. ${ }^{29}$ Upon initial cooling to $10 \mathrm{~K}$, IR bands were observed at 3252 and $3417 \mathrm{~cm}^{-1}$, which we have reassigned to the bonded $\mathrm{OH}$ stretching modes in D3 and D6, respectively. However, these peaks were absent following annealing at temperatures above $20 \mathrm{~K}$ due to a rearrangement of the metastable dimers to the lowest energy dimer, D1. This indicates that, at the initial matrix temperature of $10 \mathrm{~K}$, isomerization pathways from D3 and D6 to lower energy structures have been frozen out.

\subsection{Relevance to the condensed phases}

One of the dimers observed in this work, D3, is of particular interest because it possesses the same bonding motif as the dimeric unit of the catemer structure in crystalline AA (see Figures 1(b) and 
(c)). Although the hydrogen bonds are known to be slightly shorter in the crystal, ${ }^{48,49}$ reliable experimental data on the isolated dimer provides a suitable starting point for developing theoretical models of the crystal structure, as well as for modelling the early stages of crystal growth. As such, our experimental value of $3292 \mathrm{~cm}^{-1}$ for the bonded $\mathrm{OH}$ stretching vibration in D3 can be used as a benchmark value to test theoretical models on a simple dimeric system, before the calculations are extended to longer sections of catemer chains through to the full 3D crystalline structure. In a similar manner, our benchmark data can be used to test models of short catemer chains that are thought to exist in the liquid phase.

\section{Conclusions}

The IR spectrum of AA dimers in helium nanodroplets is dramatically different from that recorded in the gas phase. In the gas phase, the $\mathrm{OH}$ stretching region shows a very broad spectral absorption extending over many hundreds of $\mathrm{cm}^{-1}$, which originates from the cyclic dimer, the global minimum energy structure. In helium nanodroplets this broad structure disappears and is replaced by a simple spectrum consisting of a small number of relatively sharp bands. This demonstrates that metastable dimer structures completely dominate in superfluid helium and, with the aid of $a b$ initio calculations, we have been able to identify the specific isomers. Agreement between theory and experiment for the band positions is excellent.

The formation of metastable AA dimers has provided access to parts of the intermolecular potential energy surface that have been little studied experimentally. This is of particular importance because one of the observed isomers resembles the basic dimeric unit of the catemer structure found in crystalline AA, as a consequence of their identical hydrogen bonding motifs. The spectra recorded in this study can therefore serve as a benchmark for understanding hydrogen bonding interactions in the solid and liquid phases of small carboxylic acids.

\section{Conflicts of interest}


There are no conflicts to declare.

\section{Acknowledgements}

The authors wish to thank the Leverhulme Trust for providing financial support (grant number RPG-2016-308) for this work.

\section{References}

1) G. Wohlfahrt, Analysis of pH-Dependent Elements in Proteins: Geometry and Properties of Pairs of Hydrogen-Bonded Carboxylic Acid Side-Chains, Proteins 2005, 58, 396-406.

2) L, D’Ascenzo, P. A. Auffinger, P. A Comprehensive Classification and Nomenclature of Carboxyl-Carboxyl(ate) Supramolecular Motifs and Related Catemers: Implications for Biomolecular Systems, Acta Cryst. 2015, B71, 164-175.

3) O. A. Mostovaya, M. N. Agafonova, A. V. Galukhin, B. I. Khayrutdinov, D. Islamov, O. N. Kataeva, I. S. Antipin, A. I. Konovalov, I. I. Stoikov, Phosphorylated Amino Derivatives of Thiacalix[4]arene as Membrane Carriers: Synthesis and Host-Guest Molecular Recognition of Amino, Hydroxy and Dicarboxylic Acids, J. Phys. Org. Chem. 2014, 27, 57-65.

4) S. Goswami, K; Ghosh, S. Dasgupta, Molecular Recognition: Connection and Disconnection of Hydrogen Bonds, a Case Study with Dimeric and Highly Associated Monocarboxylic Acids with Simple Receptors, Tetrahedron 1996, 52, 12223-12232.

5) Y. He, B. Zhu, Y. Inoue, Hydrogen Bonds in Polymer Blends, Prog. Polym. Sci. 2004, 29, 1021-1051.

6) J. L. Derissen, A Reinvestigation of the Molecular Structure of Acetic Acid Monomer and Dimer by Gas Electron Diffraction, J. Mol. Struct. 1971, 7, 67-80. 
7) J. Chocholousova, J. Vacek, P. Hobza, Acetic Acid Dimer in the Gas Phase, Nonpolar Solvent, Microhydrated Environment, and Dilute and Concentrated Acetic Acid: Ab Initio Quantum Chemical and Molecular Dynamics Simulations, J. Phys. Chem. A 2003, 107, 3086-3092.

8) L. Turi, J. J. Dannenberg, Molecular Orbital Study of Crystalline Acetic Acid. 2. Aggregates in One, Two, and Three Dimensions, J. Am. Chem. Soc. 1994, 116, 8714-8721.

9) C. Rovira, J. J. Novoa, First-Principles Computation of the Low-Energy Polymorphic Forms of the Acetic Acid Crystal. A Test of the Atom-Atom Force Field Predictions. J. Phys. Chem. $B, 2001, \mathbf{1 0 5}, 1710-1719$.

10) H. Bertagnolli, The Structure of Liquid Acetic Acid - An Interpretation of Neutron Diffraction Results by Geometrical Models, Chem. Phys. Lett., 1982, 93, 287-292.

11) I. A. Heisler, K. Mazur, S. Yamagushi, K. Tominaga, S. R. Meech, Measuring Acetic Acid Dimer Modes by Ultrafast Time-Domain Raman Spectroscopy, Phys. Chem. Chem. Phys., 2011, 13, 15573-15579.

12) T. Nakabayashi, K. Kosugi, N. Nishi, Liquid Structure of Acetic Acid Studied by Raman Spectroscopy and Ab Initio Molecular Orbital Calculations, J. Phys. Chem. A, 1999, 103, $8595-8603$.

13) S. Imberti, D. T. Bowron, Formic and Acetic Acid Aggregation in the Liquid State, J. Phys.: Condens. Matter, 2010, 22, 404212.

14) N. B. Zineb, F. Hammami, S. Nasr, X-ray Scattering Study of Liquid Acetic Acid: Is the Short Crystal Structure Reproduced in the Liquid? J. Mol. Struc., 2010, 984, 170-175.

15) G. Desiraju, Crystal Engineering: From Molecule to Crystal, J. Am. Chem. Soc., 2013, 135, 9952-9967.

16) E. T. J. Nibbering, T. Elsaesser, Ultrafast Vibrational Dynamics of Hydrogen Bonds in the Condensed Phase, Chem. Rev., 2004, 104, 1887-1914.

17) A. V. Iogansen, Direct Proportionality of the Hydrogen Bonding Energy and the Intensification of the Stretching $v(\mathrm{XH})$ Vibration in Infrared Spectra, Spectrochim. Acta A, 1999, 55, 1585-1612. 
18) H. T. Flakus, B. Hachula, The Source of Similarity of the IR Spectra of Acetic Acid in the Liquid and Solid-state Phases, Vib. Spectrosc., 2011, 56, 170-176.

19) T. Häber, U. Schmitt, C. Emmeluth, M. A. Suhm, Ragout-jet FTIR Spectroscopy of Cluster Isomerism and Cluster Dynamics: From Carboxylic Acid Dimers to $\mathrm{N}_{2} \mathrm{O}$ Nanoparticles, Faraday Discuss., 2001, 118, 331-359.

20) C. Emmeluth, M. A. Suhm, A Chemical Approach towards the Spectroscopy of Carboxylic Acid Dimer Isomerism, Phys. Chem. Chem. Phys., 2003, 5, 3094-3099.

21) Y. J. Hu, H. B. Fu, E. R. Bernstein, IR plus Vacuum Ultraviolet Spectroscopy of Neutral and Ionic Organic Acid Molecules and Clusters: Acetic Acid, J. Chem. Phys., 2006, 125, 184308.

22) M. Goubet, P. Soulard, O. Pirali, P. Asselin, F. Réal, S. Gruet, T. Huet, P. Roy, R. Georges, Standard Free Energy of the Equilibrium Between the trans-monomer and the Cyclic-dimer of Acetic Acid in the Gas Phase from Infrared Spectroscopy, Phys. Chem. Chem. Phys., 2015, 17, 7477-7488.

23) C. Emmeluth, M. A. Suhm, D. Luckhaus, A Monomers-in-dimers Model for Carboxylic Acid Dimers, J. Chem. Phys., 2003, 118, 2242-2255.

24) J. Dreyer, Hydrogen-bonded Acetic Acid Dimers: Anharmonic Coupling and Linear Infrared Spectra Studied with Density-Functional Theory, J. Chem. Phys., 2005, 122, 184306.

25) P. Blaise, M. J. Wojcik, O. Henri-Rousseau, Theoretical Interpretation of the Line Shape of the Gaseous Acetic Acid Cyclic Dimer, J. Chem. Phys., 2005, 122, 064306.

26) M. Petković, J. Novak, N. Došlić, Shaping the Infrared Spectrum of the Acetic Acid Dimer in the OH-Stretching Range: Multiple Conformers and Anharmonic Coupling, Chem. Phys. Lett., 2009, 474, 248-252.

27) S. Oswald, E. Meyer, M. A. Suhm, Dinitrogen as a Sensor for Metastable Carboxylic Acid Dimers and a Weak Hydrogen Bond Benchmarking Tool, J. Phys. Chem. A, 2018, 122, 29332946.

28) Y. Grenie, J. -C. Cornut, J. C. Lassegues, Infrared Spectra of Matrix Isolated Acetic Acid Dimers, J. Chem. Phys., 1971, 55, 5844-5846. 
29) W. Sander, M. Gantenberg, Aggregation of Acetic and Propionic Acid in Argon Matrices - A Matrix Isolation and Computational Study, Spectrochim. Acta A, 2005, 62, 902-909.

30) S. Lopes, A. V. Domanskaya, M. Räsänen, L. Khriachtchev, R. Fausto, Acetic Acid Dimers in a Nitrogen Matrix: Observation of Structures Containing the Higher-Energy Conformer, $J$. Chem. Phys., 2015, 143, 104307.

31) K. Nauta, R. E. Miller, Formation of Cyclic Water Hexamer in Liquid Helium: The Smallest Piece of Ice, Science, 2000, 287, 293-295.

32) K. Nauta, R. E. Miller, Nonequilibrium Self-Assembly of Long Chains of Polar Molecules in Superfluid Helium, Science, 1999, 283, 1895-1897.

33) J. D. Pickering, B. Shepperson, L. Christiansen, H. Staplefeld, Femtosecond Laser Induced Coulomb Explosion Imaging of Aligned OCS Oligomers inside Helium Nanodroplets, $J$. Chem. Phys., 2018, 149, 154306.

34) J. P. Toennies, A. F. Vilesov, Superfluid Helium Droplets: A Uniquely Cold Nanomatrix for Molecules and Molecular Complexes, Angew. Chemie Int. Ed., 2004, 43, 2622-2648.

35) F. Madeja, M. Havenith, K. Nauta, R. E. Miller, J. Chocholousova, P. Hobza, Polar Isomer of Formic Acid Dimers Formed in Helium Nanodroplets, J. Chem. Phys., 2004, 120, 1055410560 .

36) M. Gantenberg, M. Halupka, W. Sander, Dimerization of Formic Acid - An Example of a "Noncovalent" Reaction Mechanism, Chem. Eur. J., 2000, 6, 1865-1869.

37) J. Tandy, C. Feng, A. Boatwright, G. Sarma, A. M. Sadoon, A. Shirley, N. D. N. Rodrigues, E. M. Cunningham, S. Yang, A. M. Ellis, Infrared Spectroscopy of Salt-Water Complexes, $J$. Chem. Phys. 2016, 144, 121103.

38) A. M. Sadoon, G. Sarma, E. M. Cunningham, J. Tandy, M. W. D. Hanson-Heine, N. A. Besley, S. Yang, A. M. Ellis, Infrared Spectroscopy of $\mathrm{NaCl}\left(\mathrm{CH}_{3} \mathrm{OH}\right)_{n}$ Complexes in Helium Nanodroplets, J. Phys. Chem. A, 2016, 120, 8085-8092.

39) M. I. Sulaiman, S. Yang, A. M. Ellis, Infrared Spectroscopy of Methanol and Methanol/Water Clusters in Helium Nanodroplets: The OH Stretching Region, J. Phys. Chem. A, 2017, 121, $771-776$. 
40) Y. Shao, Z. Gan, E. Epifanovsky, A. T. B. Gilbert, M. Wormit, J. Kussmann, A. W. Lange, A. Behn, J. Deng, X. Feng, et al., Advances in molecular quantum chemistry contained in the Q-Chem 4 program package, Mol. Phys., 2015, 113, 184-215.

41) T. Shimanouchi, Tables of Molecular Vibrational Frequencies; Consolidated Vol. I, NSRDSNBS 39; National Bureau of Standards: Washington, DC, 1972.

42) M. W. D. Hanson-Heine, M. W. George, N. A. Besley, Investigating the Calculation of Anharmonic Vibrational Frequencies Using Force Fields Derived from Density Functional Theory, J. Phys. Chem. A, 2012, 116, 4417-4425.

43) R. L. Jacobsen, R. D. Johnson III, K. K. Irikura, R. N. Kacker, Anharmonic vibrational frequency calculations are not worthwhile for small basis sets, J. Chem. Theory Comput., 2013, 9, 951-954.

44) M. L. Senent, Ab Initio Determination of the Torsional Spectra of Acetic Acid, Mol. Phys., 2001, 99, 1311-1321.

45) H. Do, N. A. Besley, Structural optimization of molecular clusters with density functional theory combined with basin hopping, J. Chem. Phys., 2012, 137, 134106.

46) D. D. Tommaso, K. L. Watson, Density Functional Theory Study of the Oligomerization of Carboxylic Acids, J. Phys. Chem. A, 2014, 118, 11098-11113.

47) A. Tkatchenko, R. A. DiStasio Jr, M. Head-Gordon, M. Scheffler, Dispersion-corrected Møller-Plesset second-order perturbation theory, J. Chem. Phys., 2009, 131, $094106 .$.

48) C. R. Groom, I. J. Bruno, M. P. Lightfoot, S.C. Ward, The Cambridge Structural Database. Acta Cryst., 2016, B72, 171-179.

49) P. -G. Jönsson, Hydrogen Bond Studies. XLIV. Neutron Diffraction Study of Acetic Acid. Acta Cryst., 1971, B27, 893-898. 